\title{
Incapacidade funcional de idosos com demência
}

\author{
Tainã Alves Fagundes, Danielle Aparecida Gomes Pereira, Kátia Maria Penido Bueno, \\ Marcella Guimaráes Assis
}

Universidade Federal de Minas Gerais - UFMG, Belo Horizonte, MG, Brasil.

\begin{abstract}
Resumo: Introdução: A demência corresponde a uma das principais causas de incapacidade e dependência na velhice e pode repercutir na capacidade funcional em todas as áreas de desempenho ocupacional, incluindo as atividades básicas e instrumentais de vida diária (ABVD e AIVD), o lazer, a participação social e outras. Objetivos: Caracterizar o nível de incapacidade funcional de idosos com demência e verificar a existência de correlação entre a funcionalidade e o estágio ou tipo de demência. Método: Estudo quantitativo, exploratório, de corte transversal, com amostra constituída por 25 cuidadores de idosos com demência. Para a caracterização dos participantes foram utilizados questionários estruturados e para avaliação da incapacidade funcional, a Escala de Avaliação de Incapacidade em Demência - DAD (Disability Assessment for Dementia) foi aplicada. Resultados: Identificou-se maior incapacidade no subitem AIVD. Tal achado é compatível com a literatura que discorre sobre a hierarquização do declínio funcional em idosos, iniciando-se pelas AIVD, enquanto as ABVD permanecem preservadas por um período maior de tempo. Não houve correlação significativa entre o tipo de demência, idade ou sexo e incapacidade funcional. Verificou-se por meio do coeficiente de spearman (rho $=0,87$ ), correlação significativa de alta magnitude entre incapacidade funcional e estágio da demência ( $\mathrm{p}=0,0001)$. Conclusão: Tais achados reiteram a relevância de priorizar a detecção precoce e prevenção do declínio funcional, que representa a manifestação de vulnerabilidade entre os idosos.
\end{abstract}

Palavras-chave: Idoso, Demência, Atividades Cotidianas, Pessoas com Deficiência.

\section{Functional disability in elderly with dementia}

\begin{abstract}
Introduction: Dementia represents one of the major causes of disability and dependence in old age and can affect functional capacity in all areas of occupational performance, including basic and instrumental activities of daily living (BADL and IADL, respectively), leisure, social participation and others. Objectives: To characterize the functional disability level in elderly people with dementia and verify the existence of correlation between functionality and the stage or type of dementia. Method: Quantitative, exploratory, cross-sectional study, with a sample of 25 caregivers of elderly with dementia. For the characterization of the participants were used structured questionnaires and to assess functional disability, the Disability Assessment Scale for Dementia - DAD was applied. Results: Greater incapacity was observed in the IADL sub item. This finding is compatible with the literature on the hierarchy in functional decline in the elderly: decline begins in IADL, while BADL remain unaffected for a longer period. There was no significant correlation between the type of dementia, age or gender and disability. It was verified through the Spearman coefficient ( $r h o=0.87$ ), a significant correlation of high magnitude between functional disability and stage of dementia $(p=0.0001)$. Conclusion: Such findings reiterate the importance of giving priority to early detection and prevention of the functional decline, which is the manifestation of vulnerability among the elderly.
\end{abstract}

Keywords: Elderly, Dementia, Everyday Activities, Disabled People.

\footnotetext{
Autor para correspondência: Marcella Guimarães Assis, Escola de Educação Física, Fisioterapia e Terapia Ocupacional, Universidade Federal de Minas Gerais, Av. Presidente Antônio Carlos, 6627, Campus Pampulha, CEP 31270-901, Belo Horizonte, MG, Brasil, e-mail: mga@ufmg.br Recebido em Mar. 31, 2016; 1ª Revisão em Jul. 7, 2016; 2ª Revisão em Set. 1, 2016; 3ª Revisão em Set. 26, 2016; Aceito em Out. 8, 2016.
} 


\section{Introdução}

Do ponto de vista demográfico, a proporção de idosos na população em geral tem crescido, principalmente devido à redução significativa das taxas de mortalidade por doenças infectocontagiosas e crônicas, ao avanço da tecnologia médica, ao maior acesso a serviços de saúde e à melhoria da qualidade de vida (ASSIS et al., 2014; BURLÁ et al., 2013). No Brasil, esse processo acontece de forma acelerada, associado à ampliação da expectativa de vida da populaçáo e pode ocasionar o aumento da prevalência e incidência de doenças incapacitantes e de prejuízos à funcionalidade (NOGUEIRA, 2010).

Dentre as doenças incapacitantes de caráter crônico-degenerativo, encontram-se as demências, que podem ser definidas como síndromes que acometem o funcionamento cerebral de forma geral, afetando funçôes como a memória, raciocínio, orientação, compreensão, cálculo, capacidade de aprendizagem, linguagem e julgamento. Pode ocorrer também comprometimento do humor e do comportamento social (BURLÁ et al., 2013; WORLD..., 2012). Simultaneamente, é necessário que os sintomas cognitivos e/ou neuropsiquiátricos acometam a habilidade de trabalho ou de realizar atividades usuais e desencadeiem declínio em relação a níveis prévios de funcionamento e desempenho, para que seja estabelecido o diagnóstico de demência (FROTA et al., 2011).

A síndrome demencial, cuja prevalência aumenta com a idade, corresponde a uma das principais causas de incapacidade e dependência na velhice, ocasionando a necessidade de cuidados constantes durante o curso da doença. O tipo predominante de demência é a Doença de Alzheimer (DA) correspondendo de 60\% a $70 \%$ dos casos, seguindo-se da Demência Vascular (DV), Demência por Corpos de Lewy (DCL) e Demência Frontotemporal (DFT) (WORLD..., 2012; BURLÁ et al., 2013).

O comprometimento cognitivo pode interferir na habilidade do indivíduo para compreender e integrar as informaçóes, etapas necessárias para a realização das atividades de vida diária. Dessa forma, o idoso com demência pode apresentar maior dificuldade em tomar decisões, planejar e desempenhar tarefas individuais, associar informaçôes, entre outras. As alterações das funções cognitivas também se relacionam com a restrição da participação social, o que pode favorecer situaçóes de isolamento e depressão (PURSER et al., 2005).

Dessa forma, a disfunçáo cognitiva pode repercutir negativamente na capacidade funcional em todas as áreas de desempenho ocupacional do indivíduo, incluindo as atividades básicas de vida diária (ABVD), como tomar banho, vestir-se e alimentar-se, as atividades instrumentais de vida diária (AIVD), como administrar os medicamentos, pagar contas e realizar tarefas domésticas, o descanso e sono, a educação, o trabalho, o brincar (no caso das crianças), o lazer e a participação social (AMERICAN..., 2015).

Os idosos que apresentam declínio funcional comumente necessitam de cuidados de terceiros. Esses cuidados podem ser classificados em informais ou formais, sendo o informal prestado pelas famílias, amigos e/ou vizinhos, e o formal ofertado por profissionais especializados (CAMARANO; MELLO, 2010). Segundo Giacomin, Uchoa e Lima-Costa (2005), os cuidadores informais emergem de relaçôes familiares quase sempre fragilizadas pela presença da doença, exigindo diversos arranjos na organização e dinâmica familiar para corresponder às necessidades da pessoa dependente. Por ocorrer em continuidade a relaçóes pessoais e familiares, frequentemente os cuidadores se veem diante de sentimentos conflitantes em relação ao alvo dos cuidados, aos familiares, às tarefas de cuidado e a si mesmos (NERI, 2010). Estudos apontam consequências negativas do cuidado para a saúde física e mental dos cuidadores, como desequilíbrio entre atividade e repouso, e estratégias de enfrentamento individual comprometidas, sendo que o tempo, em anos, que o cuidador exerce a função de cuidar está associado ao nível de impacto (FERNANDES; GARCIA, 2009; GARRIDO; MENEZES, 2004).

A Classificação Internacional de Funcionalidade, Incapacidade e Saúde (CIF), criada pela Organização Mundial de Saúde (OMS), correlaciona a funcionalidade e a incapacidade com as condiçóes de saúde, considerando as funções dos órgãos ou sistemas e estruturas do corpo, as atividades e a participaçáo social no ambiente onde a pessoa vive. Além disso, possibilita a identificação do que uma pessoa é capaz ou não de realizar na sua vida diária (FARIAS; BUCHALLA, 2005).

Em relação aos conceitos de funcionalidade e incapacidade, tem-se que funcionalidade abrange os aspectos positivos ou facilitadores da interaçáo entre um indivíduo e os seus fatores contextuais, levando em consideração as funções e estruturas do corpo, atividades e participação. Por outro lado, o termo incapacidade refere-se às deficiências ou alteraçóes das funçôes e estruturas do corpo, às limitaçôes das atividades, às restriçôes da participaçáo e à magnitude das barreiras dos fatores ambientais, traduzindo-se assim nos aspectos negativos da interação entre um indivíduo e os seus fatores contextuais. Desse modo, 
a CIF compreende a incapacidade como resultado da interação de diversos fatores e não como atributo pessoal (FONTES; FERNANDES; BOTELHO, 2010). Do ponto de vista epidemiológico, a incapacidade é geralmente mensurada por meio do relato de dependência total ou necessidade de auxílio para realizar as ABVD e as AIVD (FREEDMAN; MARTIN; SCHOENI, 2002).

Diversas escalas têm sido utilizadas para avaliar o desempenho de pessoas idosas nas atividades de vida diária (AVD). Dentre elas, destaca-se a Escala de Avaliação de Incapacidade em Demência (Disability Assessment for Dementia - DAD), baseada no modelo de saúde proposto pela OMS e desenvolvida por Gauthier e Gélinas, em 1994, para medir o desempenho de idosos com demência nas AVD (BAHIA et al., 2010; CARTHERY-GOULART et al., 2007; GÉLINAS et al., 1999).

A avaliação das AVD em pacientes com demência oferece vantagens como: obter informaçōes sobre condiçôes, habilidades e limitaçôes que não são comumente avaliadas na prática clínica, auxiliar na acurácia diagnóstica, avaliar o efeito de medicamentos, ajudar a determinar o prognóstico e avaliar e planejar a necessidade de cuidados. O estabelecimento de estágios de incapacidade ou gravidade da doença pode auxiliar a estimar a necessidade de suporte para as famílias, e em maior abrangência, estimar e incentivar a produção de políticas públicas sociais e de saúde, além de cuidados institucionais e de serviços (CARTHERY-GOULART et al., 2007).

Portanto, o objetivo do presente estudo foi caracterizar o nível de incapacidade funcional de idosos com demência e verificar a existência de correlação entre a funcionalidade e o estágio ou tipo de demência.

\section{Método}

Trata-se de estudo quantitativo, exploratório, de corte transversal, desenvolvido em um ambulatório do Hospital das Clínicas de Belo Horizonte, Minas Gerais.

\subsection{Amostra}

A amostra foi definida por conveniência e constituída por 25 cuidadores familiares de idosos com demência, atendidos no ambulatório. Os cuidadores deveriam assistir idosos que apresentassem as seguintes características: a) residir na comunidade e b) ter o diagnóstico de Demência de Alzheimer, Demência Vascular, Demência Mista, Demência
Frontotemporal ou Demência por Corpos de Lewy, segundo o Manual Diagnóstico e Estatístico das Doenças Mentais - DSM V (AMERICAN..., 2014). Foram excluídos idosos com déficits motores primários e/ou transtornos psiquiátricos prévios.

Este estudo está em conformidade com as normas internacionais para pesquisas com seres humanos e obteve aprovação do Comitê de Ética em Pesquisa da instituição (protocolo 334/06). Todos os cuidadores participantes foram orientados quanto ao objetivo do estudo e assinaram o Termo de Consentimento Livre e Esclarecido (TCLE).

\subsection{Instrumentos da coleta de dados}

Para a caracterizaçáo dos idosos e cuidadores foram utilizados questionários estruturados, que levantaram dados sociodemográficos, como idade, sexo, escolaridade, estado civil, renda e grau de parentesco, bem como dados referentes ao tempo de acompanhamento no serviço, atendimento ou náo por outros profissionais da saúde, acesso a orientações sobre a demência, dentre outros. Os dados sobre o tipo de demência e o estágio da doença, classificado pelo Clinical Dementia Rating - CDR, foram coletados nos prontuários. O CDR avalia cognição e comportamento, além da influência das perdas cognitivas na capacidade de realizar adequadamente as atividades de vida diária. Esse instrumento está dividido em seis categorias cognitivo-comportamentais classificadas em: 0 (nenhuma alteração); 0,5 (questionável); 1 (demência leve); 2 (demência moderada); e 3 (demência grave). A classificação final do CDR é obtida pela análise dessas classificaçóes por categorias, seguindo um conjunto de regras elaboradas e validadas por Morris (1993 apud MACEDO MONTAÑO; RAMOS, 2005, p. 914)

Posteriormente, a Escala de Avaliação de Incapacidade em Demência K DAD (Disability Assessment for Dementia) foi aplicada. Todos os questionários e a DAD foram respondidos pelos cuidadores. A DAD tem como objetivos: quantificar habilidades funcionais em AVD para indivíduos com déficits cognitivos, como demência, e qualificar as dimensóes cognitivas das incapacidades nas AVD, avaliando-as no que tange a funçóes executivas, sendo elas: iniciativa, planejamento, organização e desempenho efetivo. A escala inclui a avaliação de atividades básicas e instrumentais de vida diária, além do lazer (CARTHERY-GOULART et al., 2007; GÉLINAS et al., 1999). A DAD possui 17 itens que mensuram as mudanças nas ABVD e 23 itens para AIVD e atividades de lazer. As ABVD 
são avaliadas nos subdomínios: higiene, vestir-se, continência e alimentar-se. Os subdomínios das AIVD e atividades de lazer são: preparo de refeições, uso do telefone, atividade fora de casa, finanças e correspondências, medicação e lazer e tarefas de casa (ARRIGHI et al., 2013).

Cada item da DAD deve ser respondido como "sim" (1 ponto), "não" (0 ponto) ou "não pôde fazer/nunca fez" (N/D). O "sim" indica que a pessoa desempenhou a atividade sem ajuda ou sem ser lembrada nas últimas duas semanas, mesmo que isso tenha ocorrido apenas uma vez durante esse período. $\mathrm{O}$ "não" significa que a pessoa não desempenhou a atividade sem ajuda (verbal ou física) ou sem ser lembrada. Se o item é avaliado como "N/D" porque, por exemplo, a pessoa nunca fez a atividade antes ou não teve oportunidade de realizar nas últimas duas semanas, ele não é considerado. O escore total é obtido mediante a soma de pontos de cada item e conversão dela em porcentagem em um total de $100 \%$. Os escores mais altos representam menores incapacidades nas AVD, enquanto escores baixos indicam mais disfunções (GÉLINAS; GAUTHIER, 1994).

No estudo inicial, a DAD demonstrou alto grau de concordância interna $(0,96)$, interexaminadores $(0,95)$ e teste-reteste $(0,96)$ (GÉLINAS et al., 1999). A escala foi traduzida, adaptada e validada para uso no Brasil, alcançando coeficientes de correlação de 0,929 e 0,932 nas avaliaçóes inter e intraexaminadores respectivamente. Os índices de confiabilidade também Foram elevados (Kappa $=0,72$; $\mathrm{p}<0,001$ interexaminadores e Kappa $=0,85$; $\mathrm{p}<0,001$ intraexaminadores) (BAHIA et al., 2010; CARTHERY-GOULART et al., 2007).

\subsection{Procedimentos para a coleta de dados}

Os cuidadores foram abordados antes ou após a consulta médica no ambulatório. Nesse momento foi apresentado o estudo, seus objetivos e a forma como a coleta de dados se daria. Os instrumentos foram aplicados individualmente e, em sua maioria, pessoalmente, em um único encontro. Foi necessário finalizar duas coletas por telefone, devido à restrição de tempo por parte dos participantes. As coletas levaram em média 25 minutos. A necessidade de um ambiente reservado foi respeitada. O período de coleta se estendeu de dezembro de 2014 a fevereiro de 2015, resguardando os períodos de recesso do serviço.

\subsection{Análise dos dados}

Utilizou-se estatística descritiva simples (medidas de frequência e porcentagem, tendência central - média - e variabilidade - amplitude e desvio padrão) para apresentação dos resultados referentes aos aspectos clínico-demográficos e às pontuaçóes das escalas. Foi realizado o teste de correlação de Spearman para avaliar a associaçáo entre o tipo de demência, seu estágio e o escore total da DAD (funcionalidade). Foi considerado para significância estatística um $\alpha$ de 5\%.

\section{Resultados}

A amostra total do estudo foi constituída por 25 cuidadores familiares, em sua totalidade do sexo feminino $(100 \%)$ e com idade média de 53,28 \pm 12,21 anos, sendo a idade mínima 26 e máxima 78 anos. Observa-se prevalência de cuidadoras com 50 anos ou mais (64\%). As cuidadoras idosas corresponderam a $20 \%$ da amostra. A Tabela 1 apresenta outras características demográficas das cuidadoras.

O número de cuidadoras que não exercem atividade remunerada foi predominante na amostra (60\%). As cuidadoras que possuem renda individual de um salário mínimo correspondem a $44 \%$, e de dois a cinco salários mínimos a $36 \%$. Os benefícios recebidos mais prevalentes foram aposentadoria e pensão, sendo que $8 \%$ da amostra recebem os dois concomitantemente e $56 \%$ não recebem nenhum

Tabela 1. Dados demográficos das cuidadoras.

\begin{tabular}{lc}
\hline & $\mathbf{n}(\%)$ \\
\hline Estado Civil & $6(24 \%)$ \\
Solteiro(a) & $12(48 \%)$ \\
Casado(a) & $4(16 \%)$ \\
Divorciado(a)/ & \\
Separado(a) & $3(12 \%)$ \\
Viúvo(a) & \\
Escolaridade & $2(8 \%)$ \\
Analfabeto & $9(36 \%)$ \\
Fundamental incompleto & $3(12 \%)$ \\
Fundamental completo & $1(4 \%)$ \\
Médio incompleto & $8(32 \%)$ \\
Médio completo & $1(4 \%)$ \\
Superior incompleto & $1(4 \%)$ \\
Superior completo & \\
Grau de parentesco & $2(8 \%)$ \\
Irmão(a) & $18(72 \%)$ \\
Filho(a) & $4(16 \%)$ \\
Esposo(a) & $1(4 \%)$ \\
Neto(a) &
\end{tabular}


deles. Dentre as cuidadoras que trabalham, a maioria $(36 \%)$ cuida de idosos no estágio inicial ou intermediário da demência.

Verificou-se que $80 \%$ das cuidadoras coabitam com o idoso de quem cuidam e o restante divide os cuidados com outros familiares principalmente nos períodos da manhã e tarde. Em relação ao tempo à frente dos cuidados do idoso, a maioria das cuidadoras (68\%) exerce os cuidados há um período de 1 a 5 anos. Os cuidados prestados há menos de 1 ano e há mais de 10 anos tiveram distribuição equivalente ( $12 \%$ cada). As cuidadoras que cuidam dos idosos há um período de 6 a 10 anos foram minoria, correspondendo a $8 \%$ da amostra.

Do total de cuidadoras, 56\% afirmaram que receberam orientaçóes relacionadas aos cuidados necessários para com o idoso com demência e que elas foram fornecidas prioritariamente por médicos, terapeutas ocupacionais e profissionais de unidades básicas de saúde.

A média de idade encontrada entre os idosos foi de 80,28 \pm 5,33 anos, sendo 79,72 \pm 5,92 anos para as mulheres e $81,71 \pm 3,30$ anos para os homens. A caracterização demográfica dos idosos é descrita na Tabela 2.

A maior parte dos idosos (88\%) iniciou os atendimentos no ambulatório de geriatria em um período de 1 a 5 anos e $80 \%$ de todos os idosos da amostra náo estão sendo acompanhados por outros profissionais de saúde além dos médicos. Entre os profissionais que acompanham os idosos, estão terapeutas ocupacionais, fisioterapeutas e fonoaudiólogos.

Os dados referentes aos tipos e estágio de demência apresentados pelos idosos estão descritos na Tabela 3 . Nenhum idoso da amostra possuía diagnóstico de Demência por Corpos de Lewy.

As Tabelas 4 e 5 descrevem o desempenho dos idosos em cada item da DAD, detalhando-os quanto à iniciativa, planejamento e organizaçáo, e realização efetiva dentro das ABVD, AIVD e atividades de lazer.

Dentro das ABVD, observa-se maior predomínio de respostas "sim" na iniciativa. As respostas "N/D" aparecem apenas no planejamento e organização. As ABVD que a maioria dos idosos realizou com independência foram "tirou toda a roupa" (88\%) e "tentou se vestir" (84\%). O subitem "lavou-se e secou-se" foi o único em que houve predomínio (56\%) da resposta "não", evidenciando a necessidade de auxílio para realizar essas atividades.

$\mathrm{Na}$ Tabela 5 observa-se predomínio das respostas "não", evidenciando maior grau de comprometimento nas AIVD em relação às ABVD. No item "conversou adequadamente ao telefone" - realização efetiva, houve equivalência entre as respostas "sim" e "não" (44\%).

A média do escore total da DAD foi de $42,8 \%$, sendo $0 \%$ a pontuaçáo mínima e $90,9 \%$ a pontuação máxima no teste. Em relação ao escore das ABVD, os idosos apresentaram melhor desempenho, obtendo média de $63,9 \%$, com máxima de $100 \%$. O desempenho nas AIVD foi inferior ao das ABVD, com média total correspondente a $23,9 \%$ e máxima a $81,25 \%$. Em todos os casos, a média mínima foi de $0 \%$.

Não houve correlação significativa entre o tipo de demência, idade ou sexo e incapacidade funcional, medida pelo escore total da DAD. Verificou-se por meio do coeficiente de Spearman (rho $=0,87$ ), correlação significativa de alta magnitude entre incapacidade funcional e o estágio da demência ( $\mathrm{p}=0,0001)$, como descrito na Tabela 6 .

Tabela 2. Dados demográficos dos idosos com demência assistidos pelos cuidadores.

\begin{tabular}{|c|c|c|}
\hline & $\begin{array}{c}\text { Mulheres n } \\
(\%)\end{array}$ & $\begin{array}{c}\text { Homens n } \\
(\%)\end{array}$ \\
\hline Sexo & $18(72 \%)$ & $7(28 \%)$ \\
\hline \multicolumn{3}{|l|}{ Estado Civil } \\
\hline Solteiro(a) & $4(16 \%)$ & - \\
\hline Casado(a) & $5(20 \%)$ & $5(20 \%)$ \\
\hline $\begin{array}{l}\text { Divorciado(a)/ } \\
\text { Separado(a) }\end{array}$ & - & $1(4 \%)$ \\
\hline Viúvo(a) & $9(36 \%)$ & $1(4 \%)$ \\
\hline \multicolumn{3}{|l|}{ Escolaridade } \\
\hline Analfabeto & $6(24 \%)$ & $1(4 \%)$ \\
\hline $\begin{array}{l}\text { Fundamental } \\
\text { incompleto }\end{array}$ & $11(44 \%)$ & $5(20 \%)$ \\
\hline Fundamental completo & - & $1(4 \%)$ \\
\hline Médio completo & $1(4 \%)$ & - \\
\hline
\end{tabular}

Tabela 3. Tipo e estágio da demência.

\begin{tabular}{lccc}
\hline & $\begin{array}{c}\text { Mulheres } \\
\mathbf{n}(\%)\end{array}$ & $\begin{array}{c}\text { Homens } \\
\text { n (\%) }\end{array}$ & $\begin{array}{c}\text { Total } \mathbf{n} \\
(\%)\end{array}$ \\
\hline $\begin{array}{l}\text { Tipo demência } \\
\text { Demência de }\end{array}$ & $15(60 \%)$ & $3(12 \%)$ & $18(72 \%)$ \\
$\begin{array}{l}\text { Alzheimer } \\
\text { Demência Vascular }\end{array}$ & - & $1(4 \%)$ & $1(4 \%)$ \\
$\begin{array}{l}\text { Demência Mista } \\
\text { Demência }\end{array}$ & $3(12 \%)$ & $2(8 \%)$ & $5(20 \%)$ \\
Frontotemporal & - & $1(4 \%)$ & $1(4 \%)$ \\
Estágio da & & & \\
demência & & & \\
CDR 1 & $5(20 \%)$ & $1(4 \%)$ & $6(24 \%)$ \\
CDR 1-2 & $2(8 \%)$ & - & $2(8 \%)$ \\
CDR 2 & $6(24 \%)$ & $5(20 \%)$ & $11(44 \%)$ \\
CDR 2-3 & - & $1(4 \%)$ & $1(4 \%)$ \\
CDR 3 & $5(20 \%)$ & - & $5(20 \%)$ \\
\hline
\end{tabular}


Tabela 4. Desempenho dos idosos nas ABVD - DAD.

\begin{tabular}{|c|c|c|c|c|}
\hline & Questão & $\operatorname{Sim} n(\%) *$ & Não n $(\%)^{*}$ & $\mathrm{~N} / \mathrm{D} \mathrm{n}(\%)^{*}$ \\
\hline \multicolumn{5}{|c|}{ Higiene } \\
\hline Iniciativa & $\begin{array}{l}\text { Tentou lavar-se } \\
\text { sozinho }\end{array}$ & $15(60 \%)$ & $10(40 \%)$ & - \\
\hline Iniciativa & $\begin{array}{l}\text { Tentou escovar os } \\
\text { dentes ou dentadura }\end{array}$ & $14(56 \%)$ & $11(44 \%)$ & - \\
\hline Iniciativa & $\begin{array}{l}\text { Tentou cuidar dos } \\
\text { cabelos }\end{array}$ & $16(64 \%)$ & $9(36 \%)$ & - \\
\hline $\begin{array}{l}\text { Planejamento e } \\
\text { organização }\end{array}$ & Preparou o banho & $13(52 \%)$ & $11(44 \%)$ & $1(4 \%)$ \\
\hline Realização efetiva & Lavou-se e secou-se & $11(44 \%)$ & $14(56 \%)$ & - \\
\hline Realização efetiva & Escovou os dentes & $13(52 \%)$ & $12(48 \%)$ & - \\
\hline \multicolumn{4}{|c|}{ Vestir-se } & - \\
\hline Iniciativa & Tentou vestir-se & $21(84 \%)$ & $4(16 \%)$ & - \\
\hline $\begin{array}{l}\text { Planejamento e } \\
\text { organização }\end{array}$ & Escolheu vestuário & $11(44 \%)$ & $12(48 \%)$ & $2(8 \%)$ \\
\hline $\begin{array}{l}\text { Planejamento e } \\
\text { organização }\end{array}$ & $\begin{array}{l}\text { Vestiu-se sozinho na } \\
\text { ordem certa }\end{array}$ & $16(64 \%)$ & $9(36 \%)$ & - \\
\hline Realização efetiva & $\begin{array}{l}\text { Vestiu-se } \\
\text { completamente }\end{array}$ & $16(64 \%)$ & $9(36 \%)$ & - \\
\hline \multicolumn{3}{|c|}{ Continência } & $3(12 \%)$ & - \\
\hline Iniciativa & $\begin{array}{l}\text { Decidiu usar o } \\
\text { banheiro }\end{array}$ & $17(68 \%)$ & $8(32 \%)$ & - \\
\hline Realização efetiva & \multicolumn{3}{|l|}{ Alimentar-se } & - \\
\hline Iniciativa & $\begin{array}{l}\text { Decidiu que } \\
\text { precisava comer }\end{array}$ & $19(76 \%)$ & $6(24 \%)$ & - \\
\hline $\begin{array}{l}\text { Planejamento e } \\
\text { organização }\end{array}$ & $\begin{array}{l}\text { Escolheu os talheres } \\
\text { e temperos certos }\end{array}$ & $14(56 \%)$ & $9(36 \%)$ & $2(8 \%)$ \\
\hline Realização efetiva & $\begin{array}{l}\text { Comeu em um ritmo } \\
\text { normal }\end{array}$ & $19(76 \%)$ & $6(24 \%)$ & - \\
\hline
\end{tabular}

*porcentagem de questões respondidas como sim, não e N/D (nunca fez ou não teve oportunidade), respectivamente.

\section{Discussão}

No presente estudo, evidenciou-se totalidade da amostra de cuidadores pertencente ao sexo feminino. Dentre elas, $72 \%$ das cuidadoras eram filhas e $16 \%$ cônjuges dos idosos, sendo que $48 \%$ eram casadas. Os resultados vão ao encontro das pesquisas desenvolvidas com este público, que afirmam que historicamente a atividade de cuidar tem sido predominantemente função feminina, tanto no contexto familiar quanto no trabalho remunerado, e que além disso, em geral, os cuidados informais ofertados aos idosos com algum grau de dependência são providos pelas esposas ou filhas (CAMARANO; MELLO, 2010; MOREIRA; CALDAS, 2007; GIACOMIN; UCHOA; LIMA-COSTA, 2005). Segundo Camarano e Mello (2010), os cuidados informais domiciliares predominam em todo o mundo, correspondendo a cerca de $80 \%$ dos cuidados de idosos com perda de capacidade funcional nos países-membros da Organização para Cooperação e Desenvolvimento Econômico (OCDE).

Observou-se ainda que 64\% das cuidadoras possuem 50 anos ou mais, e que o número de cuidadoras idosas foi de $20 \%$. Esses resultados diferem do estudo de Moreira e Caldas (2007), que afirmam que na maioria das vezes, a faixa etária dos cuidadores pertence à mesma geração das pessoas de quem cuidam, ou seja, são idosos cuidando de outros idosos.

Em relaçáo ao estado civil dos idosos com demência, assistidos pelos cuidadores, 20\% dos homens são casados, enquanto $36 \%$ das mulheres são viúvas. Os dados vão ao encontro do estudo de Camarano e Mello (2010), em que elas afirmam que a maioria dos homens idosos dependentes é casada, o que não ocorre com as mulheres, que são predominantemente viúvas. Segundo as autoras, 
Tabela 5. Desempenho dos idosos nas AIVD - DAD.

\begin{tabular}{|c|c|c|c|c|}
\hline & Questão & $\operatorname{Sim} n(\%) *$ & Não n $(\%) *$ & N/D n $(\%)^{*}$ \\
\hline & Preparo da refeição & & & \\
\hline Iniciativa & $\begin{array}{l}\text { Tentou preparar uma } \\
\text { refeição }\end{array}$ & $10(40 \%)$ & $14(56 \%)$ & $1(4 \%)$ \\
\hline $\begin{array}{l}\text { Planejamento e } \\
\text { organização }\end{array}$ & Planejou uma refeição & $8(32 \%)$ & $16(64 \%)$ & $1(4 \%)$ \\
\hline \multirow[t]{2}{*}{ Realização efetiva } & $\begin{array}{l}\text { Cozinhou uma } \\
\text { refeição }\end{array}$ & $8(32 \%)$ & $16(64 \%)$ & $1(4 \%)$ \\
\hline & Uso do telefone & & & \\
\hline Iniciativa & Tentou telefonar & $2(8 \%)$ & $19(76 \%)$ & $4(16 \%)$ \\
\hline $\begin{array}{l}\text { Planejamento e } \\
\text { organização }\end{array}$ & $\begin{array}{l}\text { Achou e discou um } \\
\text { número }\end{array}$ & $2(8 \%)$ & $15(60 \%)$ & $8(32 \%)$ \\
\hline Realização efetiva & $\begin{array}{l}\text { Conversou } \\
\text { adequadamente }\end{array}$ & $11(44 \%)$ & $11(44 \%)$ & $3(12 \%)$ \\
\hline \multirow[t]{2}{*}{ Realização efetiva } & $\begin{array}{l}\text { Anotou e comunicou } \\
\text { recados }\end{array}$ & $5(20 \%)$ & $16(64 \%)$ & $4(16 \%)$ \\
\hline & Atividade fora de casa & & & \\
\hline Iniciativa & Decidiu sair & $7(28 \%)$ & $18(72 \%)$ & - \\
\hline $\begin{array}{l}\text { Planejamento e } \\
\text { organização }\end{array}$ & Organizou sua saída & $2(8 \%)$ & $22(88 \%)$ & $1(4 \%)$ \\
\hline Realização efetiva & $\begin{array}{l}\text { Saiu e encontrou um } \\
\text { destino familiar }\end{array}$ & $3(12 \%)$ & $20(80 \%)$ & $2(8 \%)$ \\
\hline Realização efetiva & $\begin{array}{l}\text { Utilizou transporte } \\
\text { com segurança }\end{array}$ & $1(4 \%)$ & $21(84 \%)$ & $3(12 \%)$ \\
\hline \multirow[t]{2}{*}{ Realização efetiva } & $\begin{array}{l}\text { Retornou da loja com } \\
\text { os itens apropriados }\end{array}$ & $1(4 \%)$ & $18(72 \%)$ & $6(24 \%)$ \\
\hline & $\begin{array}{c}\text { Finanças e } \\
\text { correspondências }\end{array}$ & & & \\
\hline Iniciativa & $\begin{array}{l}\text { Teve interesse em } \\
\text { assuntos pessoais }\end{array}$ & $10(40 \%)$ & $13(52 \%)$ & $2(8 \%)$ \\
\hline $\begin{array}{l}\text { Planejamento e } \\
\text { organização }\end{array}$ & $\begin{array}{l}\text { Organizou suas } \\
\text { finanças }\end{array}$ & - & $19(76 \%)$ & $6(24 \%)$ \\
\hline $\begin{array}{l}\text { Planejamento e } \\
\text { organização }\end{array}$ & $\begin{array}{l}\text { Organizou suas } \\
\text { correspondências }\end{array}$ & $1(4 \%)$ & $17(68 \%)$ & $7(28 \%)$ \\
\hline Realização efetiva & $\begin{array}{l}\text { Lidou com troco } \\
\text { Medicações }\end{array}$ & $3(12 \%)$ & $17(68 \%)$ & $5(20 \%)$ \\
\hline Iniciativa & $\begin{array}{l}\text { Decidiu tomar a } \\
\text { medicação }\end{array}$ & $2(8 \%)$ & $22(88 \%)$ & $1(4 \%)$ \\
\hline \multirow[t]{2}{*}{ Realização efetiva } & $\begin{array}{l}\text { Utilizou a medição } \\
\text { como prescrito }\end{array}$ & $1(4 \%)$ & $23(92 \%)$ & $1(4 \%)$ \\
\hline & $\begin{array}{c}\text { Lazer e tarefas fora } \\
\text { de casa }\end{array}$ & & & \\
\hline Iniciativa & $\begin{array}{l}\text { Teve interesse em } \\
\text { atividades de lazer }\end{array}$ & $8(32 \%)$ & $17(68 \%)$ & - \\
\hline Iniciativa & $\begin{array}{l}\text { Teve interesse em } \\
\text { atividades domésticas }\end{array}$ & $13(52 \%)$ & $12(48 \%)$ & - \\
\hline $\begin{array}{l}\text { Planejamento e } \\
\text { organização }\end{array}$ & $\begin{array}{l}\text { Planejou e organizou } \\
\text { atividades domésticas }\end{array}$ & $8(32 \%)$ & $16(64 \%)$ & $1(4 \%)$ \\
\hline Realização efetiva & $\begin{array}{l}\text { Completou atividades } \\
\text { domésticas }\end{array}$ & $7(28 \%)$ & $18(72 \%)$ & - \\
\hline Realização efetiva & $\begin{array}{l}\text { Ficou em casa sozinho } \\
\text { com segurança }\end{array}$ & $10(40 \%)$ & $11(44 \%)$ & $4(16 \%)$ \\
\hline
\end{tabular}

*porcentagem de questões respondidas como sim, não e N/D, respectivamente. 
Tabela 6. Correlação entre a incapacidade funcional e o tipo ou estágio da demência.

\begin{tabular}{llc}
\hline & & \multicolumn{1}{c}{$\begin{array}{c}\text { Incapacidade } \\
\text { Funcional (DAD) }\end{array}$} \\
\hline $\begin{array}{l}\text { Tipo de } \\
\text { demência }\end{array}$ & $\begin{array}{l}\text { Coeficiente } \\
\text { de correlação }\end{array}$ & $-0,075$ \\
& Valor de $\mathrm{p}$ & 0,722 \\
$\begin{array}{l}\text { Estágio da } \\
\text { demência }\end{array}$ & Coeficiente & $-0,866^{* *}$ \\
& de correlação & \\
& Valor de p & 0,0001 \\
\hline
\end{tabular}

$* * \mathrm{p}<0,001$.

esse quadro é vivenciado em todo mundo e ocorre devido à maior esperança de vida das mulheres e menor probabilidade de elas se casarem novamente.

Evidenciou-se no estudo que $36 \%$ das cuidadoras possuem ensino fundamental incompleto e $32 \%$ ensino médio completo. Em relação aos idosos com demência, assistidos pelos cuidadores, houve predomínio daqueles que possuem ensino fundamental incompleto, sendo $44 \%$ do sexo feminino e $20 \%$ do sexo masculino. A porcentagem de idosas analfabetas foi considerável (24\%) em relação aos homens (4\%). Este fato se justifica devido ao contexto social em que a populaçáo idosa do estudo cresceu, no qual as mulheres não possuíam participação nos locais de ensino e havia inclusive menor frequência à escola, priorizando-se o trabalho em detrimento da educação (MASTROENI et al., 2007).

Segundo o Instituto Brasileiro de Geografia e Estatística - IBGE (INSTITUTO..., 2013), no Brasil, além da baixa escolaridade, ainda há um número significativo de analfabetos, concentrado na população com idade acima de 55 anos (56\%) de acordo com dados coletados em 2012; no ano de 2002, 45\% dos analfabetos tinham essa faixa etária. Tais resultados apontam para o envelhecimento progressivo da população analfabeta, evidenciando a crescente dificuldade de alteraçáo deste quadro devido aos obstáculos de acesso ao ensino que se tornam cada vez maiores com avanço da idade, limitaçôes físicas, desmotivação, doenças, entre outros. Neri (2010) relata ainda que fatores como baixa escolaridade, idade, sexo feminino e baixa renda afetam significativamente as condiçóes de fragilidade e se correlacionam com déficit cognitivo.

Como esperado, o escore médio da DAD no subitem de ABVD foi mais alto $(63,9 \%)$ do que no subitem de AIVD (23,9\%), ou seja, os idosos apresentaram maior incapacidade para desempenhar as AIVD do que as ABVD. Mendes e Novelli (2015) apresentaram resultados semelhantes, visto que os idosos apresentaram $66,27 \%$ de independência nas ABVD e 52,18\% nas AIVD. Em estudo, Marra et al.
(2011) encontraram que o desempenho funcional nas AIVD avaliado pelo Índice de Lawton e Brody apresentou grande peso para determinar a severidade da demência, sugerindo que o desempenho das AIVD seja uma variável importante na diferenciação dos estágios da demência. Esses resultados são compatíveis com os achados da literatura que discorrem sobre a hierarquização do declínio funcional em idosos, inclusive aqueles com algum tipo de demência, afirmando que as AIVD são comprometidas nos estágios iniciais da doença, enquanto as ABVD permanecem intactas até quando o idoso apresenta comprometimentos cognitivos severos. Entretanto, sabe-se que a progressão individual da doença é variável e que a ordem de comprometimento do desempenho em diferentes tarefas, como as medidas pela $\mathrm{DAD}$, pode variar de paciente para paciente (ARRIGHI et al., 2013; TOZLU et al., 2014).

No presente estudo, os idosos apresentaram maior comprometimento (maior número de respostas "não") nos subitens: "utilizou a medicaçáo" (92\%), "organizou sua saída" (88\%), "utilizou o transporte com segurança” (84\%), "decidiu tomar medicaçáo" $(88 \%)$ e "saiu e encontrou um destino" (80\%), ou seja, em AIVD. Em concordância com o estudo, Arrighi et al. (2013) encontraram que as atividades nas quais os idosos com demência possuíam maior incapacidade eram, especificamente: cuidar das finanças, administrar as medicaçóes e realizar atividades fora de casa. De forma semelhante, Mendes e Novelli (2015) encontraram maior comprometimento nas AIVD, entretanto, com predomínio no lazer e atividades de casa $(62,67 \%)$ e no uso do telefone $(61,82 \%)$.

Hauber et al. (2014) investigaram a importância da manutenção da habilidade dos idosos com DA para desempenhar 10 atividades de vida diária correspondentes aos domínios da DAD, sob o ponto de vista de cuidadores dos Estados Unidos da América e da Alemanha. Em ambos os países, preservar as habilidades de utilizar o banheiro sem acidentes foi a mais importante, sendo a de gerenciar o dinheiro a de menor importância. Além disso, três das cinco atividades elencadas como mais importantes para se manter foram ABVD (utilizar o banheiro sem acidentes, alimentar-se e lavar e secar o corpo completamente). As quatro atividades menos importantes para os cuidadores foram AIVD (encontrar um destino familiar, conversar ao telefone, preparar uma refeição e gerenciar o dinheiro). Tais achados sugerem que os cuidadores de ambos os países estáo mais preocupados em preservar as habilidades dos idosos em desempenhar ABVD do que AIVD. Portanto, entender as preferências deles pode prover maior conhecimento acerca dos efeitos da 
DA e seus tratamentos, além de indicar a necessidade de desenvolvimento de avaliaçôes que considerem as preferências dos cuidadores. $\mathrm{Na}$ pesquisa, o motivo da preferência dos cuidadores teve relação com o sentimento de tristeza por parte dos idosos ao perderem tais habilidades e com a exigência de maior esforço por parte dos cuidadores para cuidar do paciente (HAUBER et al., 2014).

$\mathrm{O}$ fato de as AIVD serem as primeiras afetadas pela demência e possuírem relativamente menor importância para alguns cuidadores do que as ABVD, segundo estudo de Hauber et al. (2014), aponta para a necessidade de direcionamento de intervençôes referentes ao manejo das expectativas e impacto do declínio funcional nos cuidadores, bem como à manutenção das ABVD e/ou atividades que estes considerem importantes de serem preservadas. Sabe-se que menores capacidades funcionais têm sido associadas com o aumento da sobrecarga nos cuidadores de pacientes com demência (HAUBER et al., 2014).

Em relação às funçóes executivas, os achados do estudo mostraram que houve um predomínio de idosos que possuem iniciativa para realizar as ABVD. No que tange ao planejamento e organizaçáo, a maioria dos idosos não teve oportunidade ou nunca realizou tais etapas das atividades básicas de vida diária. A atividade "lavar-se e secar-se" foi a única ABVD em que a maioria (56\%) dos idosos da amostra não era capaz de realizar sozinha, correspondendo à função executiva de realizaçáo efetiva. O desempenho das AIVD apresentou-se predominantemente comprometido na amostra, tanto para iniciativa, planejamento e organização, quanto para o desempenho efetivo das tarefas. Segundo Paula e Malloy-Diniz (2013), as funçóes executivas predizem o desempenho funcional de idosos. Hauber et al. (2014) apontam ainda que não há correlação bem definida entre a habilidade para desempenhar uma atividade e sua habilidade para iniciá-la ou planejá-la.

Não foi encontrada correlaçáo entre idade ou sexo e incapacidade funcional medida por meio do escore da DAD. A hipótese levantada para justificar esse resultado refere-se à composição da amostra constituída apenas por idosos com demência, e é possível que nesse caso a condição de saúde tenha se tornado preponderante. Entretanto, na populaçáo geral de idosos estudada por Giacomin et al. (2008), a faixa etária $\geq 80$ anos apresentou forte associação com a incapacidade funcional.

Também não houve correlação entre o tipo de demência e o nível de funcionalidade dos idosos. Tal achado pode ter relaçáo com o número reduzido da amostra, bem como com a predominância de idosos com diagnóstico de DA (72\%) em relação aos outros tipos de demência. Entretanto, Bouwens, Van Heugten e Verhey (2009), utilizando outros instrumentos de avaliaçáo, encontraram correlação estatisticamente significativa entre a cognição (Mini Exame do Estado Mental - MEEM) e a funcionalidade (Blessed Dementia Scale - BDS) para a maioria dos tipos de demência, com exceção da Afasia Progressiva Primária. Esses autores encontraram maior prejuízo na funcionalidade de pacientes com demência relacionada à Doença de Parkinson (DP), seguido por pacientes com Demência Vascular, Demência de Alzheimer, Demência Frontotemporal e, por último, Afasia Progressiva Primária.

A análise estatística verificou a existência de correlação significativa de alta magnitude $(\mathrm{p}=0,0001$ para rho $=0,87)$ entre a incapacidade funcional $e$ o estágio da demência, sendo que, quanto maior o CDR, menor o escore, ou seja, quanto mais avançada a demência, maior o nível de incapacidade funcional do idoso. O estudo de Marra et al. (2011) aponta que o desempenho no Miniexame do Estado Mental (MEEM), a idade avançada e o desempenho funcional nas ABVD e AIVD tiveram associaçấo significativa com os três níveis de classificação da demência (CDR 1, 2 e 3). Entretanto, nesse estudo não houve correlação entre a progressão da doença e o nível de escolaridade, sexo ou diagnóstico de depressão. Arrighi et al. (2013) verificaram ainda correlação entre o declínio cognitivo e o funcional, indicando que a perda de um ponto no escore do MEEM foi associado a menos três pontos na DAD.

Por fim, no presente estudo, assim como relatado por Arrighi et al. (2013), os escores da DAD em idosos com estágio avançado da demência alcançaram efeitos chão, indicando a necessidade de desenvolvimento de outras medidas funcionais que possam ser úteis para caracterizar com propriedade o estado funcional de pacientes com demência avançada, além de auxiliar na decisão acerca da assistência e cuidados a serem prestados.

\section{Conclusão}

Os achados deste estudo evidenciaram a correlação entre o estágio da demência e a incapacidade funcional, confirmando a existência de declínio funcional à medida que a doença progride. Foi possível verificar ainda o processo de hierarquização desse declínio, iniciado pelo comprometimento do desempenho das AIVD, seguido pelas ABVD. Não foi observada correlação entre o tipo da demência e o nível de incapacidade funcional, tópico este que necessita de investigaçóes subsequentes, visto o impacto que pode 
causar nas intervençôes com diferentes populaçóes, além de ser ainda pouco explorado na literatura.

Diante do número significativo de idosos com incapacidade funcional, fica clara a relevância de priorizar a detecção precoce e prevenção do declínio funcional, que representa a manifestação de vulnerabilidade entre os idosos. Vale salientar ainda a necessidade de mais pesquisas referentes ao tema para possibilitar o planejamento de intervençôes direcionadas às necessidades específicas dos idosos e cuidadores, além de favorecer a produção de políticas públicas e institucionais que beneficiem este grupo.

Os resultados do estudo náo podem ser generalizados a toda população brasileira, pois ele foi constituído por uma amostra reduzida e composta apenas por idosos atendidos em um serviço regional de saúde. Evidencia-se a necessidade de desenvolver estudos populacionais com objetivos similares, a fim de verificar a possível relação entre os tipos de demência e a incapacidade funcional.

\section{Referências}

AMERICAN OCCUPATIONAL THERAPY ASSOCIATION - AOTA. Estrutura da prática da Terapia Ocupacional: domínio \& processo. Revista de Terapia Ocupacional da Universidade de São Paulo, São Paulo, v. 26, p. 1-49, 2015. Suplemento Especial.

AMERICAN PSYCHIATRIC ASSOCIATION - APA. Manual Diagnóstico e Estatístico de Transtorno - DSM-5. Porto Alegre: Artmed, 2014.

ARRIGHI, H. M. et al. Longitudinal changes in functional disability in Alzheimer's disease patients. International Psychogeriatric, Cambridge, v. 25, n. 6, p. 929937, 2013.

ASSIS, L. O. et al. Psychometric properties of the Brazilian version of Pfeffer's functional activities questionnaire. Frontiers in Aging Neuroscience, Lausanne, v. 6, p. 1-7, 2014.

BAHIA, V. S. et al. Functional disability in Alzheimer disease a validation study of the Brazilian version of the Disability Assessment for Dementia (DAD-Br). Alzheimer Disease \& Associated Disorders, Hagerstown, v. 24, n. 3, p. 291-295, 2010.

BOUWENS, S. F. M.; VAN HEUGTEN, C. M.; VERHEY, F. R. J. Association between cognition and daily life functioning in dementia subtypes. International Journal of Geriatric Psychiatry, Manchester, v. 24, n. 7, p. 764-769, 2009.

BURLÁ, C. et al. Panorama prospectivo das demências no Brasil: um enfoque demográfico. Revista Ciência \& Saúde Coletiva, Rio de Janeiro, v. 18, n. 10, p. 29492956, 2013.
CAMARANO, A. A.; MELLO, J. L. Introdução. In: INSTITUTO DE PESQUISA ECONÔMICA APLICADA - IPEA. Cuidados de longa duração para a população idosa: um novo risco social a ser assumido? Rio de Janeiro: IPEA, 2010. p. 13-37.

CARTHERY-GOULART, M. T. et al. Adaptação transcultural da Escala de Avaliação de Incapacidade em Demência (Disability Assessment for Dementia - DAD). Arquivos de Neuro-psiquiatria, São Paulo, v. 65, n. 3-B, p. 916-919, 2007.

FARIAS, N.; BUCHALLA, C. M. A Classificaçáo internacional de funcionalidade, incapacidade e saúde da Organizaçáo Mundial da Saúde: conceitos, usos e perspectivas. Revista Brasileira de Epidemiologia, Sáo Paulo, v. 8, n. 2, p. 187-193, 2005.

FERNANDES, M. G. M.; GARCIA, T. R. Atributos da tensão do cuidador familiar de idosos dependentes. Revista da Escola de Enfermagem da USP, São Paulo, v. 43, n. 4, p. 818-24, 2009.

FONTES, A. P.; FERNANDES, A. A.; BOTELHO, M. A. Funcionalidade e incapacidade: aspectos conceptuais, estruturais e de aplicação da Classificação Internacional de Funcionalidade, Incapacidade e Saúde (CIF). Revista Portuguesa de Saúde Pública, Lisboa, v. 28, n. 2, p. 171-178, 2010.

FREEDMAN, V. A.; MARTIN, L. G.; SCHOENI, R. F. Recent trends in disability and functioning among older adults in the United States: a systematic review. Journal of the American Medical Association, Chicago, v. 288, n. 24, p. 3137-3146, 2002

FROTA, N. A. F. et al. Critérios para o diagnóstico de doença de Alzheimer. Dementia \& Neuropsychologia, São Paulo, v. 5, p. 5-10, 2011. Suplemento Especial 1.

GARRIDO, R.; MENEZES, P. R. Impacto em cuidadores de idosos com demência atendidos em um serviço psicogeriátrico. Revista de Saúde Pública da USP, São Paulo, v. 38, n. 6, p. 835-841, 2004.

GÉLINAS, I. et al. Development of a functional measure for persons with Alzheimer's disease: the disability assessment for dementia. American Journal of Occupational Therapy, Boston, v. 53, n. 5, p. 471-481, 1999.

GÉLINAS, I.; GAUTHIER, L. Disability Assessment for Dementia (DAD): user's guide. 1994. Disponível em: <http://www.dementiaassessment.com.au/function/ dad_manual.pdf $>$. Acesso em: 04 fev. 2016.

GIACOMIN, K. C. et al. Estudo de base populacional dos fatores associados à incapacidade funcional entre idosos na Região Metropolitana de Belo Horizonte, Minas Gerais, Brasil. Cadernos de Saúde Pública, Rio de Janeiro, v. 24, n. 6, p. 1260-1270, 2008.

GIACOMIN, K. C.; UCHOA, E.; LIMA-COSTA, M. F. F. Projeto Bambuí: a experiência do cuidado domiciliário por esposas de idosos dependentes. Cadernos de Saúde Pública, Rio de Janeiro, v. 21, n. 5, p. 1509-1518, 2005. 
HAUBER, A. B. et al. Understanding the relative importance of preserving functional abilities in Alzheimer's disease in the United States and Germany. Quality of Life Research, Oxford, v. 23, n. 6, p. 1813-1821, 2014.

INSTITUTO BRASILEIRO DE GEOGRAFIA E ESTATÍSTICA - IBGE. Estudos e Pesquisas: informação demográfica e econômica. Síntese de Indicadores Sociais: uma análise das condiçôes de vida da população brasileira. Rio de Janeiro, 2013. Disponível em: <http:// biblioteca.ibge.gov.br/visualizacao/livros/liv66777.pdf>. Acesso em: 15 mar. 2016.

MACEDO MONTAÑO, M. B. M.; RAMOS, L. R. Validade da versão em português da Clinical Dementia Rating. Revista de Saúde Pública da USP, São Paulo, v. 39, n. 6, p. 912-917, 2005.

MARRA, T. A. et al. Influence of socio-demographic, clinical and functional factors on the severity of dementia. Archives of Gerontology and Geriatrics, Netherlands, v. 53, n. 2, p. 210-215, 2011.

MASTROENI, M. F. et al. Perfil demográfico de idosos da cidade de Joinville, Santa Catarina: estudo de base domiciliar. Revista Brasileira de Epidemiologia, São Paulo, v. 10, n. 2, p. 190-201, 2007.

MENDES, R. S.; NOVELLI, M. M. P. C. Perfil cognitivo e funcional de idosos moradores de uma instituição de longa permanência para idosos. Cadernos de Terapia Ocupacional da UFSCar, São Carlos, v. 23, n. 4, p. 723731, 2015.

MOREIRA, M. D.; CALDAS, C. P. A importância do cuidador no contexto da saúde do idoso. Escola Anna
Nery Revista de Enfermagem, Rio de Janeiro, v. 11, n. 3, p. 520-525, 2007.

NERI, A. L. Desafios ao bem-estar físico e enfrentados por idosos cuidadores no contexto da família: dados do fibra campinas. In: CAMARANO, A. A. (Org.). Cuidados de longa duração para a população idosa: um novo risco social a ser assumido? Rio de Janeiro: IPEA, 2010. p. 301-336.

NOGUEIRA, S. L. Fatores determinantes da capacidade funcional em idosos longevos. Revista Brasileira de Fisioterapia, São Carlos, v. 14, n. 4, p. 322-329, 2010.

PAULA, J. J.; MALLOY-DINIZ, L. F. Executive functions as predictors of functional performance in mild Alzheimer's dementia and mild cognitive impairment elderly. Estudos de Psicologia, Natal, v. 18, n. 1, p. $117-$ 124, 2013.

PURSER, J. L. et al. Mild Cognitive impairment and 10-year trajectories of disability in the Iowa established populations for epidemiologic studies of the elderly cohort. Journal of the American Geriatrics Society, Malden, v. 53, n. 11, p. 1966-1972, 2005.

TOZLU, M. D. M. et al. Functional disability in Alzheimer disease: a validation study of the turkish version of the disability assessment for dementia scale. Journal of Geriatric Psychiatry and Neurology, Thousand, v. 27, n. 4, p. 237-246, 2014.

WORLD HEALTH ORGANIZATION - WHO. Dementia: a public health priority. Genebra, 2012. Disponível em: <http://whqlibdoc.who.int/publications/2012/9789241564458_eng.pdf>. Acesso em: 30 dez. 2014 .

\section{Contribuição dos Autores}

Tainã Alves Fagundes foi responsável pela elaboração do estudo, coleta, análise descritiva dos dados e redação do texto. Marcella Guimarães Assis foi responsável pelas orientaçôes e revisão do texto. Danielle Aparecida Gomes Pereira pela análise estatística dos dados e revisão final do texto. Kátia Maria Penido Bueno pelas sugestóes e revisáo do texto. Todas as autoras aprovaram a versão final do texto.

\section{Fonte de Financiamento}

A primeira autora recebeu bolsa na Residência Integrada Multiprofissional do Hospital das Clínicas/UFMG. 